\title{
Development, remodeling and regeneration of the lung: coping with the structural and functional challenges of breathing
}

\author{
Christian Mühlfeld ${ }^{1,2,3} \cdot$ Matthias Ochs ${ }^{1,2,3}$
}

Published online: 16 January 2017

(C) Springer-Verlag Berlin Heidelberg 2016

With its vast epithelial and endothelial surface area and the extremely thin tissue barrier between the air and blood compartments, the lung is an extraordinary organ where structure and function are so closely related that any structural alterations directly affect its function as a gas exchanger. In contrast to other organs with a large surface area (such as the gut), the epithelial and endothelial surfaces are constantly and simultaneously faced with an exogenous and an endogenous milieu, which is sometimes more, sometimes less friendly. From the outside, the lungs are exposed to the air that we breathe - which besides oxygen and nitrogen may carry bacteria and viruses, particulate matter or noxious gases. From the inside, the lungs are perfused with blood that also changes its characteristics, e.g., due to inflammatory diseases or in the case of sepsis. The reaction of an individual's lung to such influences differs according to the duration of the exposure to a certain stimulus, due to the individual's age but certainly also to his or her genetic background. As the lung gains its full structural functionality only during the last weeks of fetal development, premature birth often has enormous consequences for later life. Also, genetic alterations may either directly cause lung disease (such as known for cystic fibrosis) or they may predispose for the occurrence of one of several types of lung

Christian Mühlfeld

muehlfeld.christian@mh-hannover.de

1 Hannover Medical School, Institute of Functional and Applied Anatomy, Hannover, Germany

2 Biomedical Research in Endstage and Obstructive Lung Disease Hannover (BREATH), Member of the German Center for Lung Research (DZL), Hannover, Germany

3 Cluster of Excellence REBIRTH (From Regenerative Biology to Reconstructive Therapy), Hannover, Germany disease with often unclear pathogenesis and bad prognosis due to limited therapeutic approaches. Interestingly, the patterns of lung disease-related tissue remodeling are few and depend on the mainly affected compartment, thereby defining their functional consequences: Diseases of the conducting airways (as in asthma or chronic bronchitis) cause difficulties in expiration; diseases of the vasculature lead to pulmonary hypertension and ultimately right heart failure; diffuse parenchymal diseases (e.g., pulmonary fibrosis) cause deposition of extracellular matrix and increase the gas diffusion distance; and loss of elastic properties and alveoli (as in emphysema) results in decreased gas diffusion area. Unfortunately, the endogenous regenerative potential of the lung is relatively low which means that at the moment the only life-saving option in endstage lung disease is lung transplantation. However, as in other areas of research, great efforts are currently being performed to increase lung regeneration either by stimulating the endogenous potential or by applying exogenous stem cell-based therapies.

As such, the lung is a fascinating and challenging organ for research: from a morphological point of view, it is particularly interesting because of its cellular diversity and the direct relationship between structure and function. The latter also explains why quantitative microscopic approaches (stereology) have large parts of their foundation in lung research and continue to be of enormous value. The importance of lung research is underpinned by the high prevalence and global burden of pulmonary diseases, among which asthma, emphysema, pneumonia, lung cancer and tuberculosis are the most frequent. However, less frequent diseases like pulmonary hypertension or pulmonary fibrosis are of equally great importance because of their unfavorable prognosis. Action programs at international and national level have been initiated such as the German Center for Lung Research which brings together the expertise of five German sites: Airway Research 
Center North (ARCN) located in Lübeck, Borstel and Kiel, Biomedical Research in Endstage and Obstructive Lung Disease (BREATH) in Hannover, Comprehensive Pneumology Center Munich (CPC-M), Translational Lung Research Center Heidelberg (TLRC-H), and University of Giessen and Marburg Lung Center (UGLMC).

These exciting developments in lung research make this an ideal point in time to bring together experts from various fields of lung research to provide up-to-date reviews dealing with the development, remodeling and regeneration of the lung. The series of papers is framed by two more general contributions on the structure-function relationships of the lung. In the first paper, roughly six decades after his first contribution to this journal, Ewald Weibel combines a review of his personal scientific history with a very physiological approach on the structure of the lung, thus highlighting the fruitful interaction when structure and function are considered to be two sides of the same medal rather than two separate things (Weibel 2017). The second one by John Maina builds the end point of this special issue and addresses the lung from an evolutionary point of view, emphasizing similarities and differences between vertebrate respiratory systems and their adaptation to metabolic and habitational requirements (Maina 2017).

\section{Development}

The section on development starts with a review of the morphological aspects of lung development by Johannes Schittny. This article nicely combines classical aspects of lung development with recent findings on late alveolarization and microvascular maturation, thus providing an up-to-date scholarly written piece of literature on this topic (Schittny 2017). The following paper by Veras et al. (2017) sheds light on an often neglected aspect of lung development, namely the potential interaction of the maternal environment with the prenatal lung. The authors clearly point out that maternal pre-gestational and gestational exposure to air pollution has adverse effects on lung development that sometimes last into adulthood based on maternal inflammation and fetal genetic modifications.

If fetal development is interrupted by preterm birth, the newborn's lung is faced with the dilemma that it needs to develop further while it already has to function as a gasexchanger in an immature state. In human preterm infants, this often leads to a chronic lung disease, named bronchopulmonary dysplasia. One of the reasons for this is the exposure of the lung to oxygen levels that are way above the fetal oxygen pressures to which it would normally be exposed at this developmental stage. As bronchopulmonary dysplasia has become more frequent thanks to advances in neonatal care, and consequently to the higher survival chances of very low birth weight newborns at gestational age of 24 28 weeks, animal models to study the mechanisms of this disease are of great importance and are discussed by Nardiello et al. (2017). On the opposite side of life, lung development also includes the changes that are associated with the aging of the organism. Aging involves changes of several cell biological functions at the organ level but also the exposure to a pro-inflammatory systemic milieu which makes the lung vulnerable to diseases and often worsens their outcome. The next review of this special issue deals with both these aspects, emphasizing the aging of the immune system (Brandenberger and Mühlfeld 2017).

Besides changes in the environment due to preterm birth or maternal exposure to pollutants, lung development, of course, critically depends on the coordinated developmental pattern and the existence of genes that are essential for pulmonary development. In the lung, this becomes most evident when genes encoding for proteins that are required for surfactant biosynthesis or function are affected. For example, infants born with a complete deficiency of surfactant protein B die shortly after birth due to respiratory failure. Another protein involved in innate surfactant dysfunction-associated diseases is the ATP binding cassette A3 (ABCA3), a lipid transporter located in the limiting membrane of the surfactant-storing lamellar bodies of alveolar epithelial type II cells. The consequences of genetic alterations of the gene encoding for ABCA3 range from neonatal lethality to infant or adult onset diffuse parenchymal lung diseases. Interestingly, one and the same type of mutation may cause different courses of disease. In this issue, Beers and Mulugeta (2017) provide a comprehensive and detailed review of ABCA3 biology and the consequences of its dysfunction.

\section{Remodeling}

Many lung diseases require a longstanding remodeling process until the first symptoms occur. In contrast, acute respiratory distress syndrome (ARDS) is defined on the basis of clinical criteria, namely acute onset, bilateral lung infiltrates and arterial hypoxemia. Although the pathological patternstarting with an exudative phase and leading to a fibroproliferative phase - is constant, the reasons leading to ARDS are diverse, including ischemia/reperfusion injury, pneumonia and sepsis. Despite the high mortality of ARDS, those who survive often regain normal lung function, which raises questions about the remodeling and repair processes in ARDS. This relatively novel field of research is addressed by Gill et al. (2017). As mentioned, ARDS can be the result of infectious lung disease. Understanding the interaction between various pathogens and the lung requires the use of a suitable model system. Hocke et al. (2017) discuss the advantages and pitfalls as well as the usability of ex vivo human lung tissue for a variety of bacterial and viral pathogens. Hopefully, the use of these models will not only help us 
understand the mechanisms of host defense, i.e. how the lung defends its integrity against pathogens, but also further our knowledge about the strategies pathogens use to evade the host defense. In that regard, a rather new concept of reaction to a pathogen is host tolerance, which is dealt with by Meunier et al. in this issue. Importantly, bacterial infection (even with facultative pathogens) is favored by pre-existing airway disease which is known from cystic fibrosis or chronic obstructive pulmonary disease (COPD). A common characteristic of these diseases is the reduced clearance of mucus from the airways and the resulting airflow obstruction. In the mouse, this situation can be modeled by airway-specific overexpression of epithelial sodium channels. Zhou-Suckow et al. (2017) summarize the new insights they and others have gained using this model throughout the past years, and propose enhanced mucus clearance as a promising therapeutic strategy in lung diseases of airway obstruction by mucus.

Airway obstruction is also a classical feature of bronchial asthma. Fehrenbach et al. (2017) provide us with wellstructured analysis of the current knowledge about the airway remodeling in asthma. Besides addressing the question of how airway remodeling can be assessed in quantitative terms, they have placed emphasis on the distinction between airway remodeling as a primary event in the pathogenesis or as a secondary reaction to an inflammatory stimulus. Finally, they have provided an analysis of how these aspects can be modeled in animal models. Among the pharmaceuticals used in asthma, substances stimulating $\beta 2$-adrenergic receptors or antagonizing cholinergic muscarin-receptors are among the most frequently used agents, highlighting the important role of the nervous system of the airways in asthma. However, this fact is often neglected when only parts of the components of the airway wall (such as smooth muscle cells) and their interaction with the immune system are in focus. It is therefore fortunate that Audrit et al. (2017) provide us with a plethora of information on this topic in their article for this issue.

$\mathrm{COPD}$, one of the most common lung diseases, can be subdivided into two distinct pathological entities: chronic bronchitis and emphysema. Emphysema is commonly defined as permanent enlargement of distal gas-exchanging airspaces with destruction of alveolar walls. Although considerable research efforts have been undertaken to elucidate the pathogenesis of emphysema, we are still far from a clear and comprehensive understanding of the underlying mechanisms. Craig et al. (2017) add a new perspective to this topic by presenting an immunopathological concept of emphysema which focuses on the dysregulation of macrophage-coordinated repair mechanisms in the lung. Moreover, they provide very useful information on the modeling of emphysema in mice.

The lung's mechanical stability is provided by two main components: the continuous connective tissue network and the biophysical properties of the surfactant system.
Alterations in either the connective tissue or the surfactant system may stimulate remodeling processes eventually leading to lung fibrosis. Among the fibrotic lung disorders for which the etiology is not known, idiopathic pulmonary fibrosis (IPF) is the most common. IPF is still a disease with poor prognosis and very limited therapeutic options. Knudsen et al. (2017) provide a comprehensive review on the pathology and pathophysiology of lung fibrosis, in particular IPF. They place the surfactant-producing alveolar epithelial type II cell center stage and summarize the current evidence that lung fibrosis is not primarily a disease of excessive accumulation of extracellular matrix components but rather the consequence of alveolar epithelial injury, surfactant dysfunction, alveolar collapse and collapse induration, leading to loss of ventilated lung parenchyma and alveolar surface area.

The lung is constantly challenged by exposing its large inner surface to the environment. The occupational hazards of farming are the subject of the review by Sethi et al. (2017). They provide an overview on the contaminants that agricultural workers are exposed to and their effects on the innate immune system and the inflammatory state of the lung, with particular emphasis on Toll-like receptors and surfactant proteins.

The term pulmonary hypertension $(\mathrm{PH})$ covers a variety of diseases (with diverse pathophysiology) that cause an increase in the pulmonary arterial circulation. $\mathrm{PH}$ is associated with pathological remodeling of the pulmonary arterial tree-changes that are described in a straightforward way by Rubin Tuder in this special issue. At the end of the article, Tuder (2017) emphasizes the need for more and in particular better quantitative data on the vascular remodeling in $\mathrm{PH}$ bringing designbased stereology in the scene.

With sarcoidosis, Hu et al. (2017) have addressed a topic that somehow falls between the cracks of the usual pulmonary diseases in that this granulomatous disease is not an entity that specifically affects the lungs. However, in most cases of sarcoidosis, the lung is involved, and in the case of a progressive course may ultimately lead to lung fibrosis or PH. As the authors point out, the pathogenesis of sarcoidosis is still not clear which limits targeted therapeutic interventions. As there is no single trigger of sarcoidosis, modeling the disease in animal models is challenging, a fact that $\mathrm{Hu}$ and co-workers have taken as a motivation to provide a comprehensive overview and analysis of the currently available animal models of sarcoidosis.

Nowadays, the ultima ratio of endstage, chronic lung disease is still lung transplantation. However, transplantation of the lung continues to have worse outcomes than the transplantation of other organs. Besides a phase of primary graft failure which has benefitted strongly from perioperative management during the past two decades, the transplanted lung often undergoes a progressive remodeling process predominantly affecting small airways and finally leading to chronic allograft 
failure. Kuehnel et al. (2017) have compiled much information regarding the morphology and pathophysiology, and provide a comprehensive survey of what is known about the mechanisms of chronic allograft failure which may hopefully help understand this process and stimulate new therapeutic approaches.

\section{Regeneration}

Ideally, such new therapeutic approaches would be based on the stimulation of the lung's own regenerative potential which, however, appears to be very low. Understanding the pathways that initiate, maintain, modulate and finalize normal lung development could be the key to new regenerative strategies by reinitiating these pathways in pulmonary disease. In the first article of this section, Stabler and Morrisey (2017) therefore analyze the developmental pathways and their occurrence during pulmonary repair as well as methods to model these pathways. Interestingly, when parts of the lung are removed from the body, the remaining parts of the lung are capable of performing a regenerative growth behavior which is termed compensatory lung growth. Based on her own work of the last two decades, Connie Hsia has addressed this topic and gives an overview on the mechanisms of compensatory lung growth, its limitations and its potential as a stimulus for regenerative medicine. In contrast to the common mechanistic concepts solely based on molecular aspects, Hsia's discussion highlights the importance of mechanical stimuli and structure-function relationships governing compensatory lung growth (Hsia 2017). One of the cells that may be critically involved in the signaling of mechanical stimuli in the lung resides in the interstitium of the alveolar septa: various subsets of fibroblasts which are important for the mechanical properties of the gas-exchange region. Septal fibroblasts are essential alveolar components during development and maintenance of alveolar structures and during repair phases after injury, and have therefore gained considerable interest in recent years. In this issue, Stephen McGowan gives a remarkably detailed and clear survey on the identity, function and potential of this cell type (McGowan 2017). He also does not refrain from clearly naming the gaps of knowledge that need to be filled for a fuller understanding of the fibroblasts.

As mentioned earlier, surfactant is essential for normal lung function. It is synthesized, stored, secreted and partly recycled by alveolar epithelial type II cells. The second major player in surfactant metabolism is the alveolar macrophage, and normal surfactant metabolism requires not only the function of each of these cell types but also their proper interaction. Besides acute diseases of surfactant dysfunction, the disturbance of this system leads to a variety of chronic diseases for which the restoration of the dysfunctional cell types by functioning cells may become a novel and successful therapeutic approach. Lopez-Rodriguez and co-workers have provided a short, but detailed, wrap-up about surfactant biology and the promising evidence of cell therapy by alveolar epithelial type II cells and alveolar macrophages (Lopez-Rodriguez et al. 2017).

The section on lung regeneration ends with an article addressing one of the hottest topics not only in lung research but probably in all areas of biomedical research; nevertheless, it is also one of those topics that have generated great hopes and even greater promises that still need to be fulfilled: stem cells. O'Reilly and Thébaud (2017) discuss the potential application of stem cells in the context of bronchopulmonary dysplasia. In fact, as bronchopulmonary dysplasia is a disease caused by disruption of normal development, it is also a disease of perturbed stem cell function which may predispose bronchopulmonary dysplasia as an ideal target of stem cell therapy.

We would like to thank Klaus Unsicker, Managing Editor, and Baljit Singh, Section Editor, for their initiative to invite us to guest edit a special issue of Cell and Tissue Research. Our sincere gratitude goes to Jutta Jäger, Editorial Assistant, for handling the collection of articles so smoothly. Finally, we are very grateful to all authors for their invaluable contributions which reflect the current state of the art in the field of lung development, remodeling and regeneration.

\section{References}

Audrit KJ, Delventhal L, Aydin Ö, Nassenstein C (2017) The nervous system of the airways and its remodeling in inflammatory lung diseases. Cell Tissue Res. doi:10.1007/s00441-016-2559-7

Beers M, Mulugeta S (2017) The biology of the ABCA3 lipid transporter in lung health and disease. Cell Tissue Res. doi:10.1007/s00441016-2554-Z

Brandenberger C, Mühlfeld C (2017) Mechanisms of lung aging. Cell Tissue Res. doi:10.1007/s00441-016-2511-x

Craig JM, Scott AL, Mitzner W (2017). Immune-mediated inflammation in the pathogenesis of emphysema: insights from mouse models. Cell Tissue Res doi:10.1007/s00441-016-2567-7

Fehrenbach H, Wagner C, Wegmann M (2017) Airway remodeling in asthma - what really matters. Cell Tissue Res. doi:10.1007 /s00441-016-2566-8

Gill SE, Yamashita CM, Veldhuizen RAW (2017) Lung remodeling associated with recovery from acute lung injury. Cell Tissue Res. doi:10.1007/s00441-016-2521-8

Hocke AC, Suttorp N, Hippenstiel S (2017) Human lung ex vivo infection models. Cell Tissue Res. doi:10.1007/s00441-016-2546-Z

Hsia CCW (2017) Comparative analysis of the mechanical signals in lung development and compensatory growth. Cell Tissue Res. doi:10.1007/s00441-016-2558-8

Hu Y, Yibrehu B, Zabini D, Kuebler WM (2017) Animal models of sarcoidosis. Cell Tissue Res. doi:10.1007/s00441-016-2526-3

Knudsen L, Ruppert C, Ochs M (2017) Tissue remodelling in pulmonary fibrosis. Cell Tissue Res. doi:10.1007/s00441-016-2543-2 
Kuehnel M, Maegel L, Vogel-Claussen J, Robertus JL, Jonigk D (2017) Airway remodelling in the transplanted lung. Cell Tissue Res. doi:10.1007/s00441-016-2529-0

Lopez-Rodriguez E, Gay-Jordi G, Mucci A, Lachmann N, SerranoMollar A (2017) Lung surfactant metabolism: early in life, early in disease and target in cell therapy. Cell Tissue Res. doi:10.1007 /s00441-016-2520-9

Maina JN (2017) Critical appraisal of some factors pertinent to the functional designs of the gas exchangers. Cell Tissue Res. doi:10.1007 /s00441-016-2549-9

McGowan S (2017) Understanding the developmental pathways pulmonary fibroblasts may follow during alveolar regeneration. Cell Tissue Res. doi:10.1007/s00441-016-2542-3

Meunier I, Kaufmann E, Downey J, Divangahi M (2017) Unravelling the networks dictating host resistance versus tolerance during pulmonary infections. Cell Tissue Res. doi:10.1007/s00441-017-2572-5

Nardiello C, Mižíková I, Morty RE (2017) Looking ahead: where to next for animal models of bronchopulmonary dysplasia? Cell Tissue Res. doi:10.1007/s00441-016-2534-3
O’Reilly M, Thébaud B (2017) Cell-based therapies for neonatal lung disease. Cell Tissue Res. doi:10.1007/s00441-016-2517-4

Schittny JC (2017) Development of the lung. Cell Tissue Res. doi:10.1007/s00441-016-2545-0

Sethi RS, Schneberger D, Charavaryamath C, Singh B (2017) Pulmonary innate inflammatory responses to agricultural occupational contaminants. Cell Tissue Res. doi:10.1007/s00441-017-2573-4

Stabler CT, Morrisey EE (2017) Developmental pathways in lung regeneration. Cell Tissue Res. doi:10.1007/s00441-016-2537-0

Tuder RM (2017) Pulmonary vascular remodeling in pulmonary hypertension. Cell Tissue Res. doi:10.1007/s00441-016-2539-y

Veras MM, de Oliveira Alves N, Fajersztajn L, Saldiva P (2017) Before the first breath: prenatal exposures to air pollution and lung development. Cell Tissue Res. doi:10.1007/s00441-016-2509-4

Weibel ER (2017) Lung morphometry: the link between structure and function. Cell Tissue Res. doi:10.1007/s00441-016-2541-4

Zhou-Suckow Z, Duerr J, Hagner M, Agrawal R, Mall MA (2017) Airway mucus, inflammation and remodeling: emerging links in the pathogenesis of chronic lung diseases. Cell Tissue Res. doi:10.1007/s00441-016-2562-z 